\title{
Diagnostic value of DWI-MRI for the detection of acute plaques in the relapse phase of multiple sclerosis
}

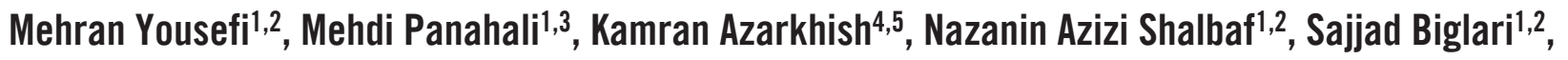 \\ Zahra Jor-Ahmad', ${ }^{1,}$, Mehdi Maghbooli ${ }^{1,2}$ \\ ${ }^{1}$ Neurology, Zanjan University of Medical Sciences, Iran \\ ${ }^{2}$ Neurology Department, Vali-e-Asr Hospital, Iran \\ ${ }^{3}$ Neurology Department, Vali-e-Asr University Hospital, Iran \\ ${ }^{4}$ Radiology, Zanjan University of Medical Sciences, Iran \\ ${ }^{5}$ Radiology Department, Vali-e-Asr Hospital, Iran
}

\begin{abstract}
Background. The 2010 revision of the McDonald criteria, widely used for the diagnosis of multiple sclerosis (MS), has established that dissemination in time (DIT) can be demonstrated by the simultaneous presence of asymptomatic gadolinium-enhancing and non-enhancing plaques on a single magnetic resonance imaging (MRI). When the use of gadolinium contrast agents is contraindicated, diffusion-weighted imaging (DWI) is utilized to confirm diffusion alterations in active inflammatory plaques. This study intended to examine whether DWI can be a viable alternative to contrast-enhanced T1-weighted imaging for demonstrating DIT in MS.

Material and methods.We assessed 30 previously diagnosed MS patients during acute relapse (based on the 2010 McDonald criteria) and evaluated their brain MRI via DWI, contrast-enhanced T1-weighted imaging, and FLAIR sequences. Asymptomatic plaques were defined as either hyperintense or non-hyperintense in DWI and enhancing or non-enhancing in T1GAD-MRI. Statistical indices for the prediction of plaque enhancement in T1 GAD-MRI via DWI-MRI were calculated and compared.

Results. The 30 participants in our study had a total of 925 demyelinating plaques that were larger than $3 \mathrm{~mm}$ in size and presented to be hyperintense in FLAIR-MRI. Diffusion hyperintensity and plaque enhancement were significantly correlated. The sensitivity, specificity, positive predictive value, negative predictive value, and accuracy of DWI were calculated to be $69.66 \%, 99.76 \%, 96.88 \%, 96.86 \%$, and $96.86 \%$, respectively.

Conclusions. Hyperintense DWI findings do not necessarily overlap with contrast enhancements in T1 GAD-MRI. DWI was shown to produce a higher rate of false-positive results. Our study concludes that although T1 GAD-MRI should not be replaced by DWI to determine DIT due to its lower specificity, DWI's continued use as a surrogate screening imaging sequence whenever the use of T1GAD-MRI is of concern is not without its merits.
\end{abstract}

Keywords: multiple sclerosis, MRI, diffusion-weighted, contrast-enhanced T1- weighted

\section{INTRODUCTION}

Multiple sclerosis is a multifactorial disease that mostly involves myelin sheaths and axons of the brain and spinal cord. Though symptoms usually begin in early adulthood, most of the patients have an average life expectancy. Still, the disease's significant comorbidities impose hefty moral and economic expenses to the patient and health ser- vices. Diagnostic criteria for multiple sclerosis include clinical and paraclinical findings and emphasizes this principle that dissemination in space (DIS) and dissemination in time (DIT) of the plaques should be proven, and other differential diagnoses are excluded $(1,2)$. The wrong diagnosis seriously results in neglecting and inappropriate treatment of the disease. DWI-MRI is a technique based on the free passage of water molecules in the 
tissue (3). Free movement of water molecules in a particular direction, for example, in brain white matter tracts, is called diffusion (4). Among the many pathologic processes result in diffusion interruption, ischemic stroke is the most common one, which causes cellular edema due to $\mathrm{Na}-\mathrm{K}$ ATPase pumps dysfunction. Diminished intercellular space results in water molecules' limitation free movement, which appears hyper-intense in DWI-MRI (5). DWI-MRI indicates a restriction of movement of water molecules in some processes such as brain abscess and hyper-cellular brain tumors; however, confirmation of real restriction of diffusion in this sequence is based on hyper-intensity of the lesion and real limitation in water molecules movement in another sequence named ADC map(6), so hyper-intensity of a plaque in both DWI-MRI and ADC map indicates that the diffusion is facilitated and not restricted (7). Therefore, ADC map sequences should also be contemplated for interpretation of hyper-intensity disclosed in DWI-MRI and differentiating the type of water molecules movement disorder (restricted or facilitated) (8).

In the acute phase of an ischemic stroke, the lesion is hyper-intense in DWI-MRI and hypo-intense in ADC map sequences because of cytotoxic edema and decreased osmosis (9). Evolution to the subacute phase (7-10 days) changes edema's nature to vasogenic type and increases the osmosis; therefore, the lesion is still hyper-intense in DWI-MRI, but it begins to switch to an iso signal lesion in ADC map sequences.

Generally, acute demyelinating plaques in MS are hyper signal in both DWI-MRI and ADC map sequences; this imaging character is related to vasogenic edema surrounding the acute plaques, typically hyper signal in ADC map sequences (10). Acute demyelinating plaques in relapsing MS may have vasogenic edema due to increased diffusion, which leads to high signal in ADC sequences (11). In contrast, acute ischemic lesions have low signal in ADC sequences due to cytotoxic edema and diffusion restriction. Acute demyelinating lesions with restricted diffusion (ADLRD) in MS were reported in some recent studies. One of these studies encompasses an evaluation of morphology, symptoms extent, signal characteristics, and changes over time on nine acute demyelinating lesions with restricted diffusion. Lesions with the described characteristics of typical plaques in T1GAD-MRI sequences of 6 patients with RRMS from two academic centers were compared with lesions shown hyper signal in DWI or ADC map sequences, and there were no significant differences between ADLRD and typical plaques (12). Periventricular and low T2 signal plaques were mostly ADLRD rather than standard plaques. Conclusively, the current study and other similar studies about ADLRD have shown that these plaques are new types of MS plaques, and their pathogenesis is still unknown.

On the other hand, the presence of mentioned plaques in brain MRI of a young adult induces difficulty in differentiating multiple sclerosis from a stroke. In some MS plaques in the hyperacute phase, there are similar features to acute stroke lesions in MRI with DWI and ADC map sequences (13). Figure 1 represents the brain MRI of a young woman with an internal capsule involvement (14). Albeit abnormal findings were similar to a stroke, finally supplemental surveys established MS diagnosis.
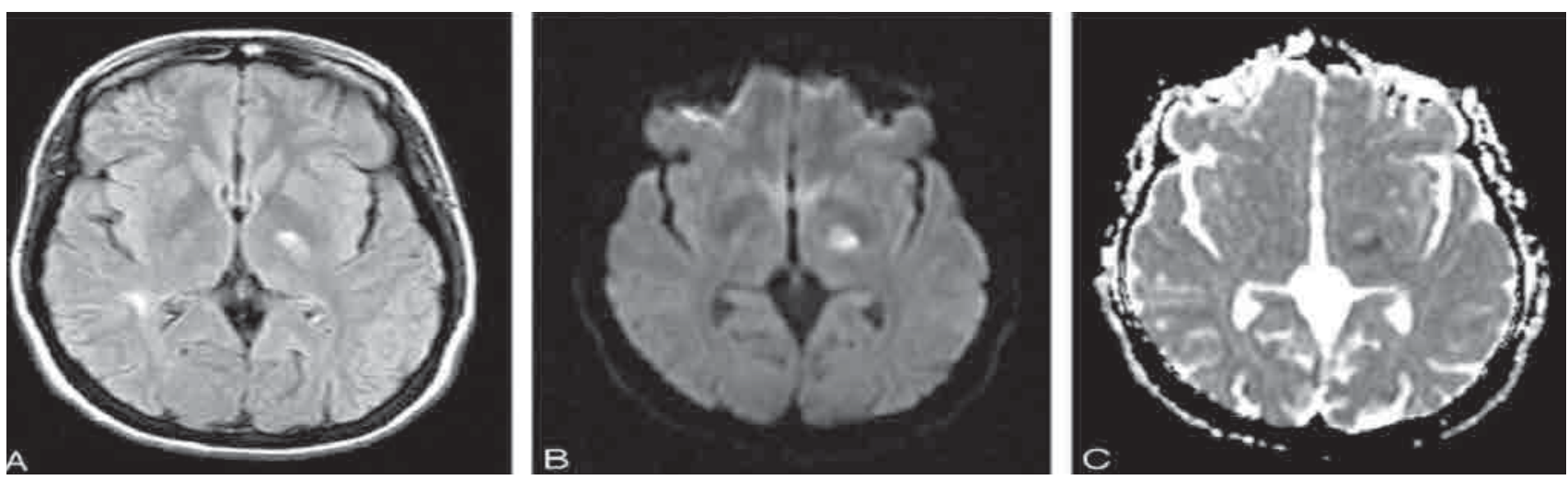

FIGURE 1. Brain MRI findings in 2 hours after stroke-like onset of focal neurologic deficits. A. FLAIR sequence, showing high signal intensity in the left internal capsule. B. DWI, showing high signal intensity in the left internal capsule. C. ADC mapping, showing reduced ADC of the left internal lesion 
The current study aimed to compare DWI-MRI sequences with T1 GAD sequences in the acute phase of multiple sclerosis relapse, especially in conditions such as pregnancy, renal failure, and hypersensitivity to contrast agents where the use of contrast agents is contraindicated and whether DWI-MRI sequences can help us to determine the acuity of plaques and modify the protocol of disease management.

\section{MATERIAL AND METHODS}

In this cross-sectional study, 30 patients within clinical relapse of multiple sclerosis underwent a brain imaging method with a contrast agent, FLAIR, and DWI-MRI in a period of 4 to 6 months. This project was accepted in the Ethics Committee of Zanjan University of medical sciences with the code of ZUMS.REC.1394.46.

All patients with an acute attack of MS (diagnosed according to the revised McDonald 2010 criteria), hospitalized in the neurology ward of Vali-e-Asr hospital in Zanjan, from April 2014 until December 2014 were entered into the study. The primary variable of this study was the number of MRI plaques, not the number of patients. Each patient had an average of 10-15 plaques in MRI.

30 patients admitted in the relapse phase of MS underwent a brain MRI with and without contrast and then with DWI sequences, before administering treatment (steroid pulse), usually within the first 24 hours of hospitalization. Patients who fulfilled the diagnostic criteria depending on MRI and clinical features were registered in the study. Patients who did not fulfill the criteria or established another diagnosis were excluded. Acute plaque numbers were calculated separately in T1GAD and DWI sequences; all substantial old and new plaques were diagnosed based on FLAIR sequences. Necessary data including whole plaque numbers and acute plaque numbers depend on T1 GAD and DWI sequences, were obtained separately.
Gathered data, including the participants' demographic characteristics and total plaque numbers and plaques distinguished in T1GAD and DWI sequences, were analyzed via SPSS16 software. The two image modalities were compared using the cross-table method. Sensitivity, specificity, PPV, NPV, and DWI accuracy were calculated using T1GAD sequences as standard, then based on McDonald 2010 criteria, a $2 \times \times 2$ table was formed (Table 1).

\section{RESULTS}

30 patients in the relapse phase of RRMS entered our study, 20 were women, and 10 were men. The participants' mean age was $32.33 \pm 9.6$ years, and most of the patients were young adults (figure 2 ). They were all indicated to receive glucocorticoid pulse therapy, however, before initiating treatment, DWI-MRI, T1GAD-MRI, and FLAIR-MRI sequences were performed, and the results were compared.

Based on FLAIR sequences, there were 925 plaques in total, between the 30 patients. Each participant's mean number of plaques was $27.8 \pm 19.5$ in FLAIR sequences, $3 \pm 3$ in T1 GAD-MRI, and $2 \pm 2$ in DWI-MRI sequences. Periventricular plaques were the most prevalent plaques found. Figure 3 represents the numbers of plaques in all three different MRI sequences for each patient.

There was a significant relationship between DWI-MRI and T1GAD-MRI sequences, and overall more plaques were enhanced in T1GAD-MRI, and their locations were consistent with the findings of DWI-MRI.

The number of patients and enhanced plaques in every sequence and their relationship were compared using the cross-table method. For example, four of the patients with five new plaques in T1GAD-MRI had four plaques in DWI-MRI, and one had five plaques. In five other patients, T1 GAD-MRI showed three plaques: one had one

TABLE 1. Definition of results according to T1GAD and DWI sequences

\begin{tabular}{|c|c|c|c|c|}
\hline & \multicolumn{2}{|l|}{ T1GAD-MRI } & \multirow[b]{2}{*}{ Total } \\
\hline & & New & Old & \\
\hline \multirow{2}{*}{$\begin{array}{l}\bar{x} \\
\sum_{1}^{1} \\
\sum_{0}\end{array}$} & New & Lesions as new Plaques in both sequences & $\begin{array}{l}\text { Plaques that were new in DWI-MRI but not in } \\
\text { T1 GAD-MRI }\end{array}$ & Total new plaques in DWI-MRI \\
\hline & Old & $\begin{array}{l}\text { Plaques that were new in T1GAD-MRI } \\
\text { but not in DWI-MRI }\end{array}$ & Lesions as previous Plaques in both sequences & Total old plaques in DWI-MRI \\
\hline & & Total new plaques in T1 GAD-MRI & Total old plaques in T1 GAD-MRI & Total plaques \\
\hline
\end{tabular}


60
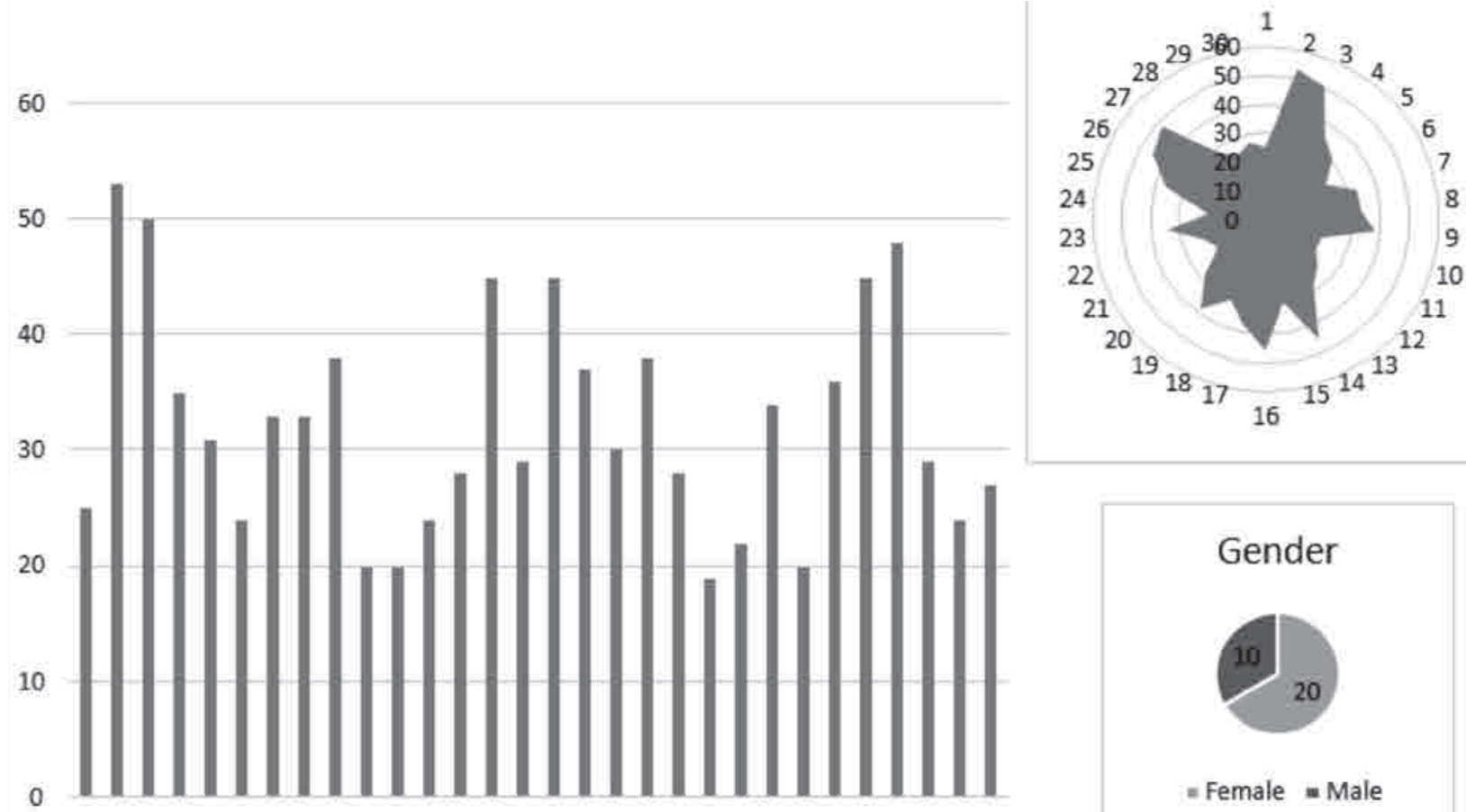

1234556789101112131415161718192021222324252627282930

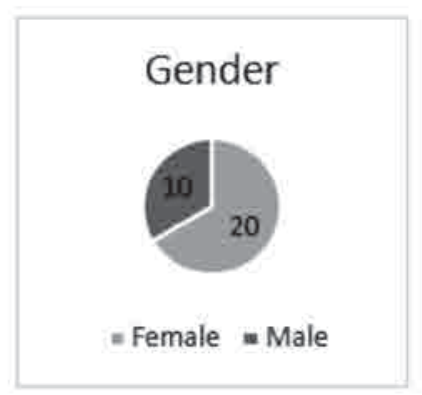

FIGURE 2. The information of the patients adopted for the experiments

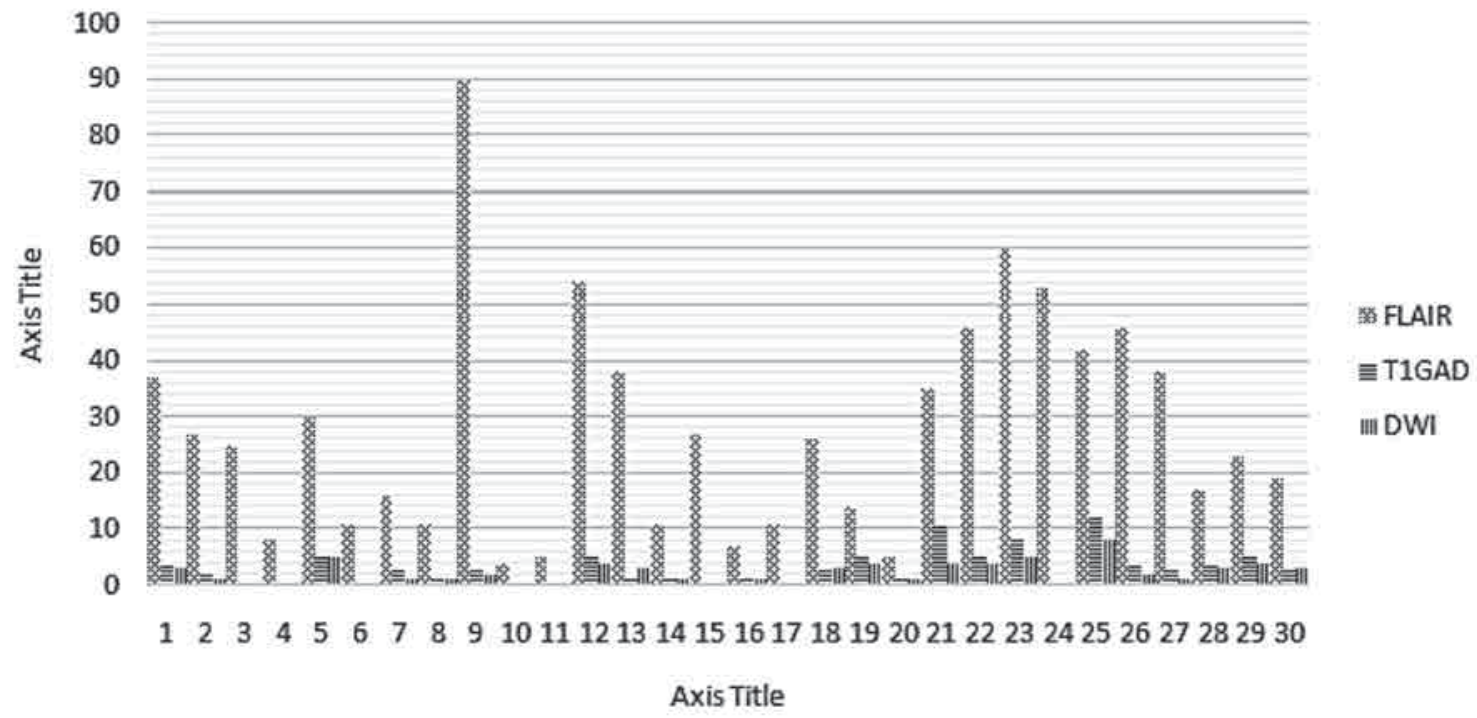

FIGURE 3. Number of plaques in 3 different MRI sequences for each patient

plaque, two patients had two plaques, and another two had three plaques in DWI-MRI sequences. None of the two types of imaging sequences revealed new plaques in 8 patients. We conclude that there should not necessarily be an association between the two modalities. Main indices, including sensitivity, specificity, PPV, NPV, and accuracy of the test, were calculated based on T1GAD-MRI being the gold standard imaging modality and were compared to DWI-MRI. We calculated both the total number of plaques and old plaques count according to FLAIR sequences and then compared them with the related sequences (table 2).

TABLE 2. Discrimination of plaques based on old or new plaques numbers

\begin{tabular}{|c|c|c|c|c|}
\hline & \multicolumn{2}{|c|}{ T1GAD-MRI } & \multirow[b]{2}{*}{ Total } \\
\hline & & New & Old & \\
\hline \multirow{2}{*}{$\begin{array}{l}\bar{x} \\
\sum_{0}^{1} \\
\sum_{0}^{1}\end{array}$} & New & 62 & 2 & 64 \\
\hline & Old & 27 & 834 & 861 \\
\hline & & 89 & 836 & 925 \\
\hline
\end{tabular}


Our final results demonstrated a sensitivity of $69.66 \%(59.46,78.24)$, a specificity of $99.76 \%$ (99.13, 99.93), a positive predictive value of $96.88 \%(89.3,99.14)$, a negative predictive value of $96.86 \%$ (95.48-97.84) and test diagnostic accuracy of $96.86 \%$ (95.53-97.81) for finding acute plaques via DWI-MRI. We used the Wilson Score for our statistical analysis.

\section{DISCUSSION}

We studied 30 patients in the relapse phase of RRMS. The disease's generic distribution was similar to the worldwide statics, and the disease affects females two times more than males. The age distribution of patients in our study was not entirely in line with patterns worldwide, mostly young adults. Older adults were also present in our study.

In 2014 an animal study about the pathology of MS plaques and their relationship with MRI sequences revealed that in the most common animal models (experimental autoimmune encephalomyelitis), plaques with contrast agent absorption in T1GAD indicate blood-brain barrier (BBB) breakage (15). Blackhole lesions on MRI imply axonal damages in pathology. Plaques with vascular changes in pathology also show changes in DWI-MRI and M.R. angiography. Indeed, different imaging protocols of MRI and their evaluation with computer-aided tools help us to understand underlying pathologies, but these methods necessitate further studies. Data analysis of a study on 22 MS patients with 384 plaques ascertained a significant relationship between the hyper signal in DWI-MRI and contrast enhancement in T1GAD sequences. Sensitivity, specificity, positive predictive value (PPV), negative predictive value (NPV) and accuracy of the study were $100 \%, 68.9 \%$, $32.3 \%, 100 \%, 72.1 \%$ with $\mathrm{P}$ value $<0.5$ respectively (16).

A study in Iran in 2016 by Davoudi Y, Foroughipour $\mathrm{M}$ indicated that while CE-MRI will depict more positive results, there are cases in which DWI will show a positive result while CE-MRI is negative (17).

Chung-Ping Lo in 2014 showed a hyperintense DWI finding does not necessarily overlap with contrast enhancement (16). There are many false positives, possibly representing other stages of le- sion development. Although DWI may not replace CE T1WI imaging to demonstrate DIT due to the low PPV, it may serve as a screening MRI sequence where the use of GBCAs is a concern.

Current study participants were often known patients with a long duration of MS and had many plaques in MRI. Indeed, patients with a prolonged disease or higher probability of attack were spontaneously recruited in the study since our research was designed for the disease's acute attack phase. As expected, the abundance distribution of plaques was higher in 3 particular brain sites, including the periventricular region, compared to juxtacortical and sub-tentorial ones. Approximately in one-third of the patients presenting with new clinical symptoms and full filling the diagnostic McDonald 2010 criteria, none of the T1GAD-MRI and DWI-MRI sequences could show new plaques. However, the acute clinical evolution of the disease was present, and the patient was receiving treatment. A comparison of two MRI sequences, DWI and T1GAD, declared a high degree of overlapping in plaques' locations. Other related studies have concluded the same result. A comparison of two MRI sequences by the cross-tabulation method did not show a complete overlapping, and most of the time, T1GAD-MRI had many plaques. The explanation of this difference could be that plaque enhancement in T1 GAD-MRI is due to blood-brain barrier breakage and its pathologic stage. In contrast, plaque enhancement in DWI-MRI resulted from vasogenic edema and inflammation $(18,19)$.

On the other hand, some false positive cases had plaque enhancement in DWI-MRI without any noticeable enhancement in T1GAD-MRI sequences. This may be because of the disease's pathological stage and the presence of cytotoxic edema or poor imaging techniques. Therefore, statistically, DWI-MRI is not a perfect alternative for T1GAD-MRI. MRI with contrast agent may reveal these plaques again in T1GAD sequences after a proper time interval. Our results showed a $69.66 \%$ CI (59.46-78.24\%) sensitivity rate for DWI-MRI. According to this low sensitivity rate, this modality has a lower potential for full filling DIT criteria than T1GAD-MRI sequences.

On the other hand, the specificity rate of $99.76 \%$ CI (99.13-99.93\%), in addition to the positive predictive value of $96.88 \%$ and negative predictive value of $96.86 \%$ and test accuracy of $96.86 \%$ for 
DWI-MRI, make it a reliable surrogate. These findings are reliable for patients described based on the McDonald 2010 criteria, along with the exclusion of other differential diagnoses. Overall, DWI-MRI is not a powerful modality for confirmation of acute plaques in MS In a proper clinical setting, the results are most reliable for demonstrating DIT criteria, especially when false positive plaque enhancements in DWI sequences are proven in different T1GAD-MRI sequences.

Conflict of interest: none declared

Financial support: none declared

\section{REFERENCES}

1. Polman $\mathrm{CH}$, Reingold SC, Brenda Banwell B, et al. Diagnostic criteria for multiple sclerosis: 2010 Revisions to the McDonald criteria. Amer Neuro Assoc. 2011;69(2):292-302.

2. Filippi M, Agosta F. Imaging biomarkers in multiple sclerosis. J Mag Reson Imag. 2010;31(4):770-788.

3. Pagani E, Bammer R, Horsfield MA, Rovaris M, Gass A, Ciccarelli O, Filippi M. Diffusion MR imaging in multiple sclerosis: technical aspects and challenges. AJNR Am J Neuroradiol. 2007 Mar;28(3):411-20.

4. Steven AJ, Zhuo J, Melhem ER. Diffusion kurtosis imaging: an emerging technique for evaluating the microstructural environment of the brain. AJR Am J Roentgenol. 2014 Jan;202(1):W26-33.

5. Radwan MEM, Aboshaera KO. One step diagnosis of multiple sclerosis disease activity, dissemination in time and space using diffusion weighted MRI. The Egyptian J Radiology Nuclear Med. 2016;47(4):1557-1563.

6. Hygino da Cruz LC Jr, Batista RR, Domingues RC, Barkhof F. Diffusion magnetic resonance imaging in multiple sclerosis. Neuroimaging Clin N Am. 2011 Feb;21(1):71-88.

7. Rueda-Lopes FC, Hygino da Cruz LC Jr, Doring TM, Gasparetto EL. Diffusion-weighted imaging and demyelinating diseases: new aspects of an old advanced sequence. AJR Am J Roentgenol. 2014 Jan;202(1):W34-42.

8. Abou Zeid N, Pirko I, Erickson B, Weigand SD, Thomsen KM, Scheithauer B, Parisi JE, Giannini C, Linbo L, Lucchinetti CF. Diffusion-weighted imaging characteristics of biopsy-proven demyelinating brain lesions. Neurology. 2012 May 22;78(21):1655-62.

9. Shen JM, Xia XW, Kang WG, Yuan JJ, Sheng L. The use of MRI apparent diffusion coefficient (ADC) in monitoring the development of brain infarction. BMC Med Imaging. 2011 Jan 6;11:2.

10. Cercignani M, lannucci G, Filippi M. Diffusion-weighted imaging in multiple sclerosis. Ital J Neurol Sci. 1999;20(5 Suppl):S246-9.

\section{CONCLUSIONS}

If DWI-MRI discloses new plaques in an MS patient, we can use this protocol for the fulfillment of DIT criteria; but two pitfalls must be considered: false-positive results were more prevalent in DWIMRI; the higher potency and specificity of T1GADMRI for demonstrating new plaques. Eventually, this study does not recommend DWI-MRI as a standard alternative for the confirmation of active MS and the establishment of DIT criteria.

11. Abolhasani Foroughi $A$, Salahi $R$, Nikseresht A, Heidari $H$, Nazeri $M$, Khorsand A. Comparison of diffusion-weighted imaging and enhanced T1-weighted sequencing in patients with multiple sclerosis. Neuroradiol J. 2017 Aug;30(4):347-351.

12. Balashov KE, Lindzen $\mathrm{E}$. Acute demyelinating lesions with restricted diffusion in multiple sclerosis. Mult Scler. 2012 Dec;18(12):1745-53.

13. Rovira A, Pericot I, Alonso J, Rio J, Grivé E, Montalban X. Serial diffusion-weighted MR imaging and proton MR spectroscopy of acute large demyelinating brain lesions: case report. AJNR Am J Neuroradiol. 2002 Jun-Jul;23(6):989-94.

14. Rosso C, Remy P, Creange A, Brugieres P, Cesaro P, Hosseini H. Diffusion-weighted MR imaging characteristics of an acute strokelike form of multiple sclerosis. AJNR Am J Neuroradiol. 2006 May;27(5):1006-8.

15. Nathoo N, Yong VW, Dunn JF. Understanding disease processes in multiple sclerosis through magnetic resonance imaging studies in animal models. Neuroimage Clin. 2014 Apr 24;4:743-56.

16. Lo CP, Kao HW, Chen SY, Chu CM, Hsu CC, Chen YC, Lin WC, Liu DW, Hsu WL. Comparison of diffusion-weighted imaging and contrast-enhanced T1-weighted imaging on a single baseline MRI for demonstrating dissemination in time in multiple sclerosis. BMC Neurol. 2014 May 7;14:100.

17. Davoudi Y, Foroughipour M, Torabi R, Layegh P, Matin N, Shoeibi A. Diffusion Weighted Imaging in Acute Attacks of Multiple Sclerosis. Iran J Radiol. 2016 Feb 15;13(2):e21740.

18. Balashov KE, Aung LL, Dhib-Jalbut S, Keller IA. Acute multiple sclerosis lesion: conversion of restricted diffusion due to vasogenic edema. J Neuroimaging. 2011 Apr;21(2):202-4.

19. Eisele P, Szabo K, Griebe M, Rossmanith C, Förster A, Hennerici M, Gass A. Reduced diffusion in a subset of acute MS lesions: a serial multiparametric MRI study. AJNR Am J Neuroradiol. 2012 Aug;33(7):1369-73. 